\title{
Corilagin induces apoptosis, autophagy and ROS generation in gastric cancer cells in vitro
}

\author{
JIAJIA XU ${ }^{1}$, GONGYE ZHANG $^{2}$, YINPING TONG ${ }^{2}$, JIAHUI YUAN ${ }^{2}$, YUANYUE LI $^{1}$ and GANG SONG ${ }^{2}$ \\ ${ }^{1}$ Fisheries College, Jimei University, Xiamen, Fujian 361021; ${ }^{2}$ Cancer Research Center, \\ Medical College of Xiamen University, Xiamen, Fujian 361102, P.R. China
}

Received July 9, 2018; Accepted December 10, 2018

DOI: $10.3892 /$ ijmm.2018.4031

\begin{abstract}
Corilagin, a unique component of the tannin family, has been identified in several medicinal plants. In previous literature, corilagin exhibited a marked anticancer property in a variety of human cancer cells. However, the biological effects of corilagin on gastric cancer and the mechanisms involved remain to be fully elucidated. In the present study, it was reported that corilagin induced inhibition of cell growth in SGC7901 and BGC823 cells in a concentration-dependent manner. It was found that corilagin exhibited less toxicity towards normal GES-1 cells. Furthermore, the study showed that corilagin induced the apoptosis of gastric cancer cells mainly via activating caspase-8, -9, -3 and poly ADP-ribose polymerase proteins. Simultaneously, it was verified that corilagin triggered autophagy in gastric cancer cells and the inhibition of autophagy improved the activity of corilagin on cell growth suppression. In addition, corilagin significantly increased intracellular reactive oxygen species production, which is important in inhibiting the growth of gastric cancer cells. Finally, it was shown that necroptosis cannot be induced by corilagin-incubation in SGC7901 and BGC823 cell lines. Consequently, these findings indicate that corilagin may be developed as a potential therapeutic drug for gastric cancer.
\end{abstract}

\section{Introduction}

Gastric cancer is a common form of malignant tumor in gastrointestinal tract cancer. In recent years, gastric cancer ranks as the third highest for mortality rate and fourth highest

Correspondence to: Mr. Yuanyue Li, Fisheries College, Jimei University, 43 Yindou Road, Jimei, Xiamen, Fujian 361021, P.R. China

E-mail: 979949223@qq.com

Mr. Gang Song, Cancer Research Center, Medical College of Xiamen University, Xiang'an South Road, Xiang'an, Xiamen, Fujian 361102, P.R. China

E-mail: gangsongsd@xmu.edu.cn

Key words: corilagin, gastric cancer, apoptosis, autophagy, reactive oxygen species for morbidity rate of all types of cancer in the world (1). In less developed countries in particular, it is the third most frequently diagnosed cancer in men and the leading cause of cancer-associated mortality (2). Although a decreasing trend in gastric cancer incidence and mortality rates has been observed in several countries, it is not effectively treated currently due to its complex etiology (3). Surgery is the optimal treatment approach for gastric cancer at present, however, the 5-year-survival rate remains low. The majority of patients relapse following surgery (4). Systemic and partial chemotherapy is necessary for patients with advanced gastric cancer (5). However, gastric cancer cells show high drug resistance to most common chemotherapeutic agents $(6,7)$. Consequently, identifying novel effective anticancer drugs is crucial for cancer therapy.

Corilagin [ $\beta$-1-O-galloyl-3,6-(R)-hexahydroxydiphenoylD-glucose] is a unique component of the tannin family. The molecular weight of corilagin is 634.45 (8). Corilagin has been identified in several medicinal plants, including Longan (9), Lumnitzera racemose (10), Terminalia catappa L (11) and Phyllanthus species (12). Previous studies have shown that corilagin has extensive pharmacological actions, including anti-inflammatory (13), antioxidative (11), antiviral (12), hepatoprotective (10), anti-atherogenic (8) and antitumor activities, and low adverse effects. A study by Guo et al (14) demonstrated that corilagin can protect against herpes simplex virus-1 (HSV-1) encephalitis through inhibiting the Toll-like receptor (TLR)2 signaling pathways. In their study, it was found that corilagin markedly prevented an increase in the levels of TLR2 and its downstream mediators following HSV-1 challenge. In addition, it was shown that corilagin directly inhibited inflammatory cytokines, including tumor necrosis factor (TNF)- $\alpha$ and interleukin (IL)- 6 proteins. The effect of corilagin on hepatoprotective properties has been reported; the underlying hepatoprotective mechanism of corilagin was examined in a trauma-hemorrhagic shock rodent model and it was found that the drug markedly alleviated pro-inflammatory cytokine and neutrophil accumulation via the AKT pathway (15). Similarly, Du et al (16) indicated that corilagin effectively relieved hepatic fibrosis by inhibiting the expression of molecules associated with the IL-13/signal transducer and activator of transcription 6 signaling pathway. Furthermore, studies have confirmed that corilagin has notable antitumor effects on a number of tumor cells, including 
hepatoma (17), ovarian cancer (18), cholangiocarcinoma (19) and glioblastoma (20). Studies have shown that corilagin can markedly inhibit the growth of ovarian cancer cells in vitro and in vivo by increasing cell cycle arrest at the G2/M stage, enhancing apoptosis and inhibiting the TGF- $\beta$ signaling pathways $(18,21,22)$. However, the mechanism involved has not been fully elucidated in gastric cancer. Therefore, the present study was designed to investigate the effect of corilagin on the apoptosis, autophagy and necroptosis of SGC7901 and BGC823 human gastric cancer cells.

Cell apoptosis, controlled by a large number of genes, acts as one of the most vital processes in the regulation of carcinogenesis (23). It has been well documented that signaling pathways leading to apoptosis involve the sequential activation of cysteine proteases, known as caspases (24). In the initial step of the apoptotic process, it triggers the activation of an apoptotic signaling program, which leads to cell death rather than killing the cell directly (25).

Autophagy, commonly referred to as 'self-eating', is sensitized by various types of intracellular stress, for example, DNA damage and low nutrient levels. Autophagy is mostly a protective process involving the capture and digestion of cellular constituents within lysosomes. However, the hyperactivation of autophagy can cause autophagic cell death (26).

Necroptosis is a more recently described form of programmed cell death, which differs from apoptosis and has similar morphological characteristics to necrosis, including cell swelling, rupture of the plasma membrane and condensation of the chromatin. In recent years, necroptosis has attracted wide attention due to its specific function in physiological and pathological processes. Receptor interaction protein 3 (RIP3), a serine/threonine kinase, is required for activation of the necrotic cell death pathway. However, RIP3 deficiency has been found in the majority of cancer cell lines. Therefore, RIP3 may be important in cancer progression $(27,28)$.

Reactive oxygen species (ROS), a cellular metabolite, is important in the development of cancer (29). Oxidative stress is an imbalance between ROS and the antioxidant defense system. Excessive ROS production at certain levels act as signal molecules to stimulate cell apoptosis and DNA damage (30). Accordingly, it is recognized that ROS are involved in antitumor function.

In the present study, the effects of corilagin-induced growth inhibitionand apoptosiswerefirstevaluatedingastriccancercells using a 3-(4,5-dimethylthiazol-2-yl)-2,5-diphenytetrazolium bromide (MTT) assay, EdU proliferation assay, lactate dehydrogenase (LDH) release assay, ROS generation assay, Hoechst 33342 staining detection, flow cytometric analysis and western blot analysis. Subsequent investigation focused on the ability of corilagin to induce autophagy in human gastric cancer cells and whether the inhibition of autophagy can enhance the effect of corilagin. Finally, experiments were performed to investigate whether necroptosis occurs in gastric cancer cells following corilagin treatment.

\section{Materials and methods}

Reagents and antibodies. Corilagin with a purity of $99.36 \%$ was purchased from Qingyun Biology (Nanjing, China). Acridine orange (AO), Earle's balanced salt solution (EBSS), dimethyl sulfoxide (DMSO), N-acetyl-L-cysteine (NAC), MTT, anti- $\beta$-actin antibody (cat. no. A1978) and Hoechst 33342 were purchased from Sigma-Aldrich; Merck KGaA (Darmstadt, Germany). Fetal bovine serum (FBS), horseradish peroxidase-conjugated goat anti-rabbit antibody (cat. no. A24537), RPMI-1640 medium and Dulbecco's modified Eagle's medium (DMEM) were purchased from Gibco; Thermo Fisher Scientific, Inc. (Waltham, MA, USA). Penicillin-streptomycin was obtained from PAA; GE Healthcare (Chicago, IL, USA). Chloroquine (CQ) and necro-sulfonamide (NSA) were purchased from Tocris Biosciences (Bristol, UK). Pancreatin was purchased from Solarbio Science \& Technology Co., Ltd. (Beijing, China). Z-VAD-FMK and necrostatin-1 (Nec-1) were obtained from Selleck Chemicals (Houston, TX, USA). Anti-LC3 antibody (cat. no. NB100-2220) was purchased from Novus Biologicals, LLC (Littleton, CO, USA) and anti-RIP3 antibody (cat. no. GTX107574) was obtained from Genetex Inc. (Irvine, CA, USA). The lactate dehydrogenase (LDH) cytotoxicity assay kit, anti-caspase-3 antibody (cat. no. AC030), anti-caspase- 8 antibody (cat. no. AC056), anti-caspase-9 antibody (cat. no. AC062) and anti-poly ADP-ribose polymerase (PARP) antibody (cat. no. AP102) were purchased from Beyotime Institute of Biotechnology (Haimen, China). The Annexin V-FITC/propidium iodide (PI) apoptosis detection kit and ROS detection kit were obtained from Nanjing KeyGen Biotech Co., Ltd. (Nanjing, China). All other chemicals were of analytical grade available from commercial sources.

Cell culture. The SGC7901 and BGC823 human gastric cancer cell lines were obtained from the Chinese Academy of Sciences (Shanghai, China). The GES-1 human gastric mucosal epithelial cell line, HeLa human cervical cancer cell line and HT-29 human colorectal carcinoma cell line were donated by the School of Life Sciences, Xiamen University (Xiamen, China). The HT-29, SGC7901 and BGC823 cells were maintained in RPMI-1640 medium, the HeLa and GES-1 cells were cultured in DMEM. The media were supplemented with $10 \%$ FBS and $100 \mathrm{U} / \mathrm{ml}$ penicillin/streptomycin. The cells were cultured at $37^{\circ} \mathrm{C}$ in a humidified atmosphere of $95 \%$ air and $5 \% \mathrm{CO}_{2}$.

Cell viability assay. The cell growth was measured using an MTT assay. The cells were seeded into 96-well plates at a density of $1 \times 10^{4}$ cells/well. After $24 \mathrm{~h}$ of incubation, the cells were treated with corilagin at serial concentrations $(0,10,20$, $30,40$ and $50 \mu \mathrm{M})$ for $24 \mathrm{~h}$. Furthermore, for synergistic effect analysis, corilagin $(30 \mu \mathrm{M})$ combined with Z-VAD-FMK (CQ, NAC, NSA or Nec-1) were added to the cells and incubated for $24 \mathrm{~h}$. At the end of treatment, $20 \mu \mathrm{l}$ of MTT was added to each well (final concentration: $0.5 \mathrm{mg} / \mathrm{ml}$ ) and incubated for $4 \mathrm{~h}$. The medium was then replaced with $150 \mu \mathrm{l}$ of DMSO. The absorbance was measured with a microplate reader at a wavelength of $490 \mathrm{~nm}$.

EdU proliferation assay. The cells (density, $1 \times 10^{4}$ cells/well) were seeded into a 96 -well plate and then treated with corilagin for $24 \mathrm{~h}$. Cell proliferation was assessed using the EdU proliferation assay kit (Guangzhou RiboBio Co., Ltd., Guangzhou, China). The stained cells were observed under a fluorescence microscope (Olympus IX51; Olympus Corporation, Tokyo, Japan). 
Cell morphological analysis using Hoechst 33342 staining. The cells (density, $1.5 \times 10^{5}$ cells/well) were cultured in 12-well plates and treated with corilagin $(0,10,20$ and $30 \mu \mathrm{M})$ for $24 \mathrm{~h}$. Following treatment, the cells were washed twice with PBS and fixed with $4 \%$ paraformaldehyde (Sigma-Aldrich; Merck KGaA) in PBS for $10 \mathrm{~min}$ at room temperature. The cells were then washed twice with PBS and stained with $10 \mu \mathrm{g} / \mathrm{ml}$ Hoechst 33342 for $15 \mathrm{~min}$ at $37^{\circ} \mathrm{C}$. The morphological changes were detected using a fluorescence microscope (Olympus Corporation). This assay was performed following the procedures as described by Lee et al (31).

$L D H$ release assay. The cells (density, $1 \times 10^{4}$ cells/well) were seeded into 96-well plates and then treated with corilagin at different concentrations $(0,10,20$ and $30 \mu \mathrm{M})$. The LDH assay was then performed according to the steps described by the manufacturer's protocol for the LDH cytotoxicity assay kit (Beyotime Institute of Biotechnology).

Annexin V-FITC/PI double staining apoptosis detection. The Annexin V-FITC/PI kit was used to detect the apoptosis of cells by flow cytometry. Briefly, the cells were cultured and treated with corilagin $(0,10,20$ and $30 \mu \mathrm{M})$. After $24 \mathrm{~h}$, the cells were collected and harvested. The cells were then washed with cold PBS, adjusted to $1 \times 10^{6}$ cells $/ \mathrm{ml}$ in $1 \mathrm{X}$ binding buffer and stained with Annexin V-FITC and PI solution for $15 \mathrm{~min}$ at room temperature in the dark. Finally, the stained cells were analyzed by flow cytometry (Beckman Coulter, Inc., Brea, CA, USA).

Acridine orange (AO) staining analysis. The SGC7901 and BGC823 cells were collected and plated into 12-well plates. After $12 \mathrm{~h}$ of incubation, the cells were treated with corilagin for $24 \mathrm{~h}$. The cells were then stained with $1 \mu \mathrm{M} \mathrm{AO}$ at $37^{\circ} \mathrm{C}$ for $15 \mathrm{~min}$. Finally, the acidic autophagic vacuoles were visualized using a fluorescence microscope.

Detection of ROS generation assay. The ROS generation assay was performed as previously described (32). The SGC7901 and BGC823 cells were exposed to various concentrations $(0,10,20$ and $30 \mu \mathrm{M}$ ) of corilagin with or without NAC for $24 \mathrm{~h}$, washed with PBS, and resuspended in culture medium (without serum) containing $10 \mu \mathrm{M}$ DCFH-DA. As positive control, the cells were treated with Rosup (50 $\mu \mathrm{g} / \mathrm{ml})$ for $30 \mathrm{~min}$. Subsequently, ROS production was detected by flow cytometry.

Reverse transcription-polymerase chain reaction (RT-PCR) assay. Total RNA from the gastric cancer cells was extracted using TRIzol reagent (Invitrogen; Thermo Fisher Scientific, Inc.), following which cDNA was synthesized with $1 \mu \mathrm{g}$ of total RNA at $37^{\circ} \mathrm{C}$ for 15 min using the Primescript RT reagent kit (Takara Biotechnology Co., Ltd., Dalian, China). The RT-PCR assay was performed using the Roche LightCycler ${ }^{\circledR} 96$ Real-time PCR system with the SYBR Premix EX Taq II kit (Takara, Biotechnology Co., Ltd.). The reaction volume was $20 \mu \mathrm{l}$, including SYBR Premix EX Taq II $(10 \mu \mathrm{l})$, Forward Primer $(0.8 \mu \mathrm{l})$, Reverse Primer $(0.8 \mu \mathrm{l})$, cDNA $(2 \mu \mathrm{l})$, Sterilized Purified Water $(6.4 \mu \mathrm{l})$. The amplification conditions were the following: Initial pre-degeneration at $95^{\circ} \mathrm{C}$ for $2 \mathrm{~min}$, followed by 40 cycles of denaturation at $95^{\circ} \mathrm{C}$ for $10 \mathrm{sec}$ and annealing/extension at $60^{\circ} \mathrm{C}$ for $20 \mathrm{sec}$. The relative mRNA levels were determined with the $2^{-\Delta \Delta \mathrm{Cq}}$ method (33). GAPDH was used as an RIP3 mRNA internal control. The RIP3 primers were forward, 5'-ACTCCCGGCTTAGAAGGACT-3' and reverse, 5'-GCCCTGCTCCTCTTGGTAAG-3'. The GAPDH primers were forward, 5'-TGCACCACCAACTGCTTAGC-3' and reverse, 5'-GGCATGGACTGTGGTCATGAG-3'.

Western blot analysis. Following corilagin treatment, the cells were collected and lysed in RIPA lysis buffer. The protein quantity was analyzed using a Bicinchoninic acid protein assay kit (Thermo Fisher Scientific, Inc.). The protein samples $(20 \mu \mathrm{g})$ were separated using SDS-PAGE (8-12\%) and transferred onto PVDF membranes. Subsequently, these membranes were blocked using 5\% non-fat milk, and incubated with the appropriate primary antibodies against LC3 (cat. no. NB100-2220), RIP3 (cat. no. GTX107574), caspase-3 (cat. no. AC030), caspase-8 (cat. no. AC056), caspase-9 (cat. no. AC062) and PARP (cat. no. AP102; all 1:1,000) at $4^{\circ} \mathrm{C}$ overnight. The membranes were then incubated with horseradish peroxidase-conjugated goat anti-rabbit secondary antibody (1:5,000; cat. no. A24537) for $1 \mathrm{~h}$ at room temperature. Finally, the protein bands were visualized using the ECL detection system (Pierce; Thermo Fisher Scientific, Inc.).

Statistical analysis. All data are presented as the mean \pm standard deviation of three independent experiments. Statistical analysis was performed using Student's t-test with GraphPad Prism 6 software (GraphPad Software, Inc., La Jolla, CA, USA). $\mathrm{P}<0.05$ was considered to indicate a statistically significant difference.

\section{Results}

Corilagin inhibits human gastric cancer cell growth but has no effect on normal cells. To evaluate the growth inhibitory effect of corilagin (Fig. 1A), the effects of corilagin on the proliferative activities of the cells were investigated. The effect of corilagin on the growth of GES-1 human gastric mucosal epithelial cells was examined using an MTT assay. As shown in Fig. 1B, the cell viability in the corilagin-treated groups did not decrease. The effects on SGC7901 and BGC823 cell lines were also investigated using the MTT assay. As presented in Fig. 1C, corilagin significantly inhibited cell proliferation of the two cancer cell lines in a concentration-dependent manner compared with the control group. The cytotoxic effect of corilagin on SGC7901 and BGC823 cells was also assessed by microscopic observations. The results (Fig. 1D) indicated that the cells exposed in corilagin for $24 \mathrm{~h}$ exhibited morphological changes compared with the untreated cells. Specifically, the cell number was effectively decreased and the cells became rounded following corilagin treatment, and this occurred in in a dose-dependent manner. The results of the EdU assay (Fig. 2A and B) confirmed the effects on the proliferation of SGC7901 and BGC823 cells. Based on these preliminary results, $0,10,20$ and $30 \mu \mathrm{M}$ were selected as the appropriate concentration ranges for SGC7901 and BGC823 cells in the following experiments. 
A

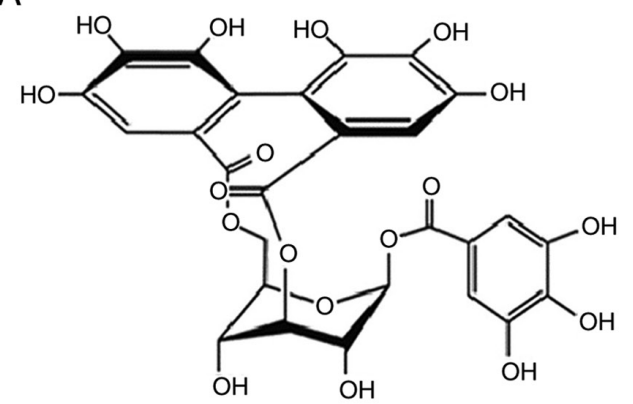

C

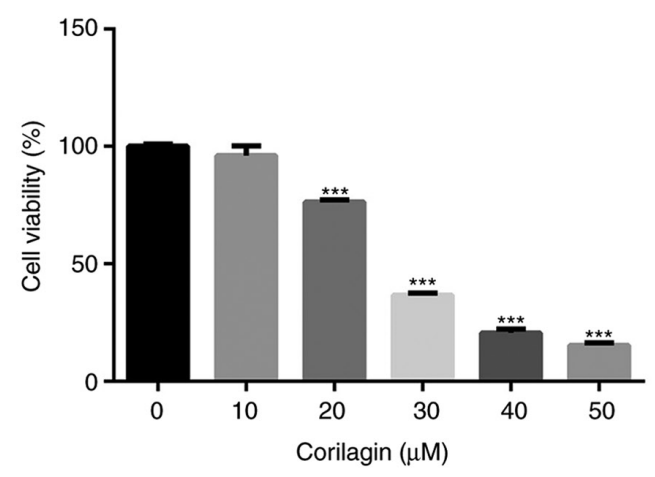

D

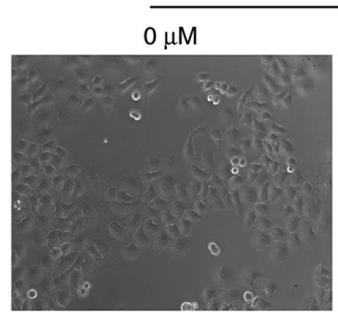

$20 \mu \mathrm{M}$

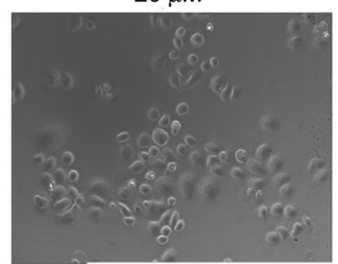

$40 \mu \mathrm{M}$

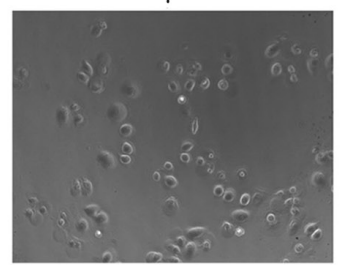

B

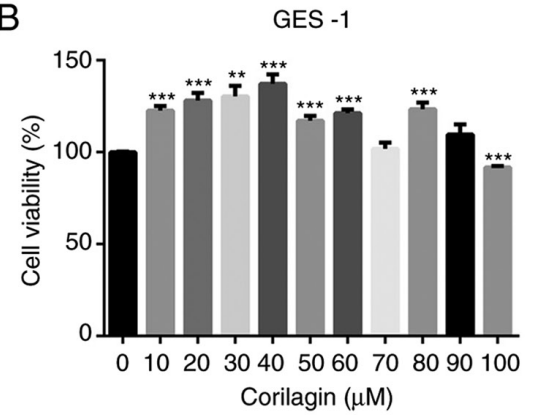

BGC823

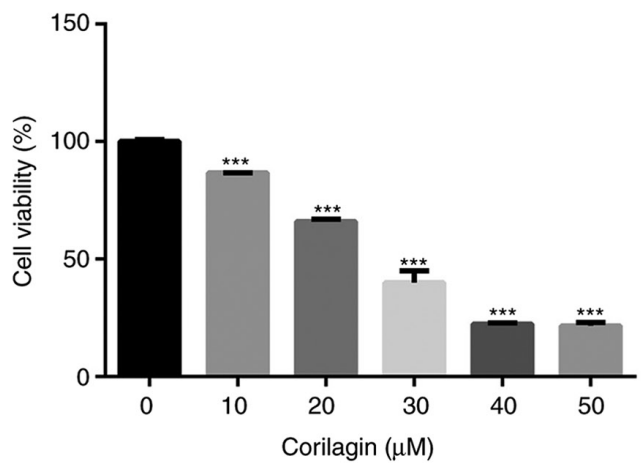

BGC823

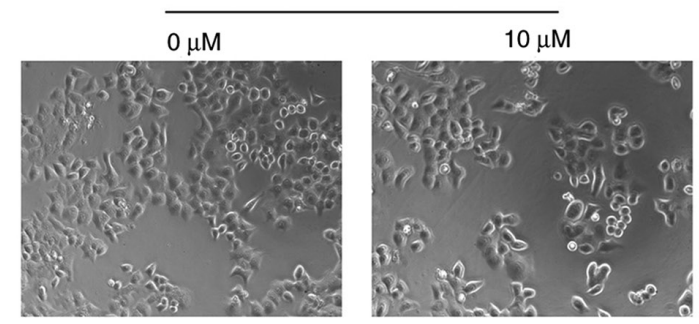

$20 \mu \mathrm{M}$

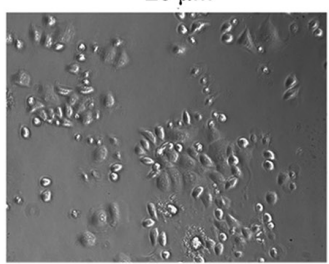

$40 \mu \mathrm{M}$

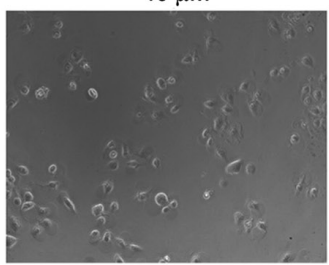

$30 \mu \mathrm{M}$

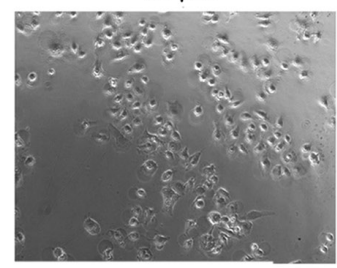

$50 \mu \mathrm{M}$

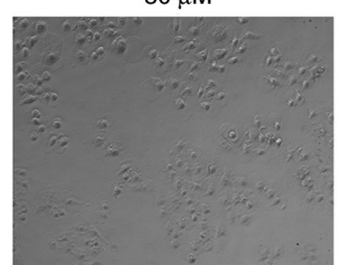

Figure 1. Effect of corilagin on the viabilities of human gastric cancer cells. (A) Chemical structure of corilagin. (B) GES-1 cells were treated with various concentrations of corilagin $(0-100 \mu \mathrm{M})$ for $24 \mathrm{~h}$. (C) SGC7901 and BGC823 cells were treated with different concentrations of corilagin (0, 10, 20, 30, 40 and $50 \mu \mathrm{M}$ ) for $24 \mathrm{~h}$. The cell viabilities were examined using a 3-(4,5-dimethylthiazol-2-yl)-2,5-diphenytetrazolium bromide assay. (D) SGC7901 and BGC823 cells were exposed to corilagin at different concentrations $(0-50 \mu \mathrm{M})$ for $24 \mathrm{~h}$, and images were captured by microscopy at magnification, $\mathrm{x} 200$. Data are reported as the mean \pm standard deviation $(n \geq 3)$ of three replicate experiments. Significant differences from the control group were measured using Student's t-test. ${ }^{* *} \mathrm{P}<0.01,{ }^{* * * *} \mathrm{P}<0.001$.

Corilagin induces the apoptosis of SGC7901 and BGC823 cells. To determine whether the efficacy of corilagin on cell growth inhibition was a result of apoptosis. Annexin V/PI and Hoechst 33342 staining assays were combined to assess apoptotic cell death. First, the morphological characteristics were observed in apoptotic cells and the results are shown in Fig. 3A. The untreated control cells manifested weak homogeneous blue fluorescence and a normal nuclear structure. The corilagin-treated cells exhibited chromosomal condensation, formation of apoptotic bodies and brighter granular 
A
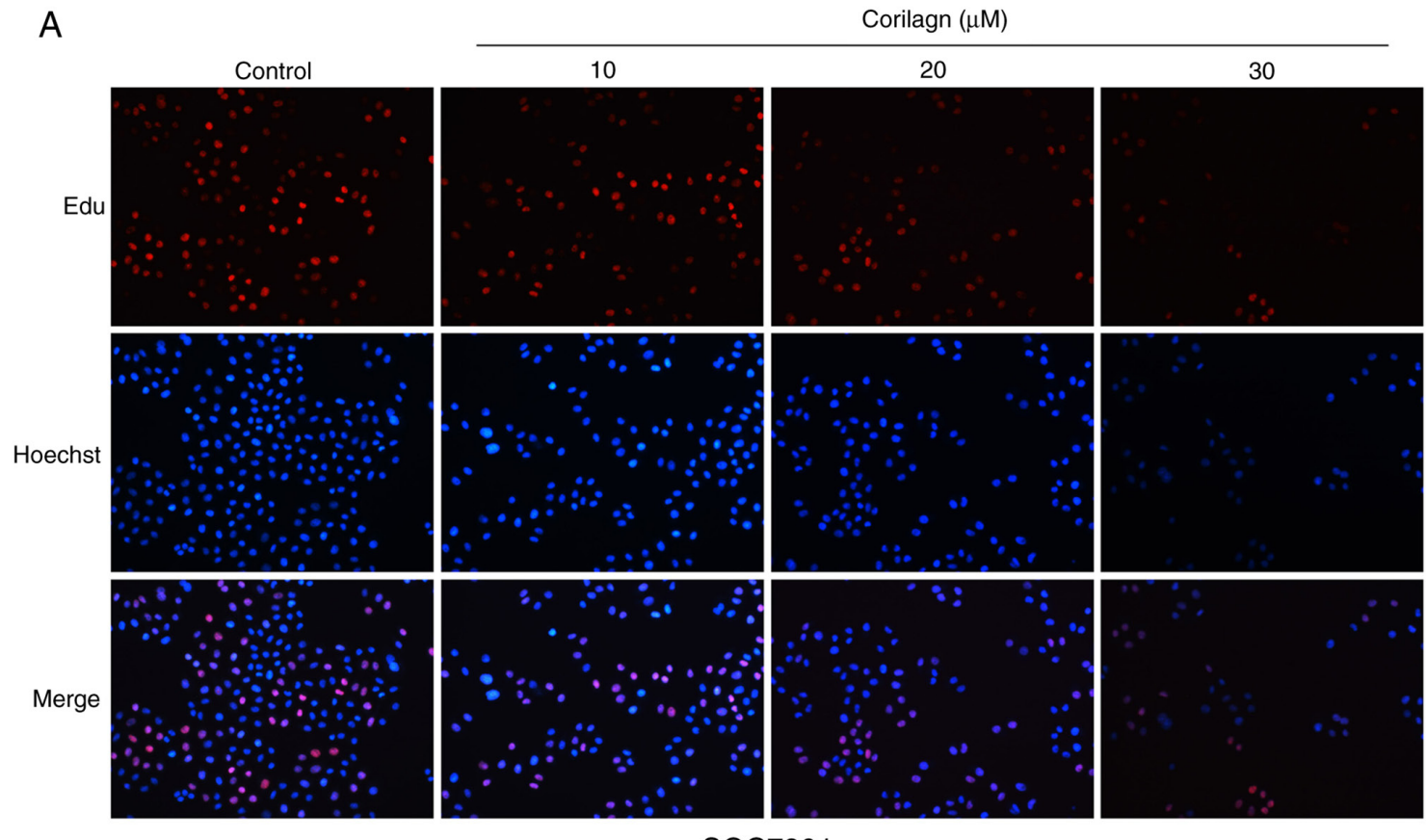

SGC7901

B

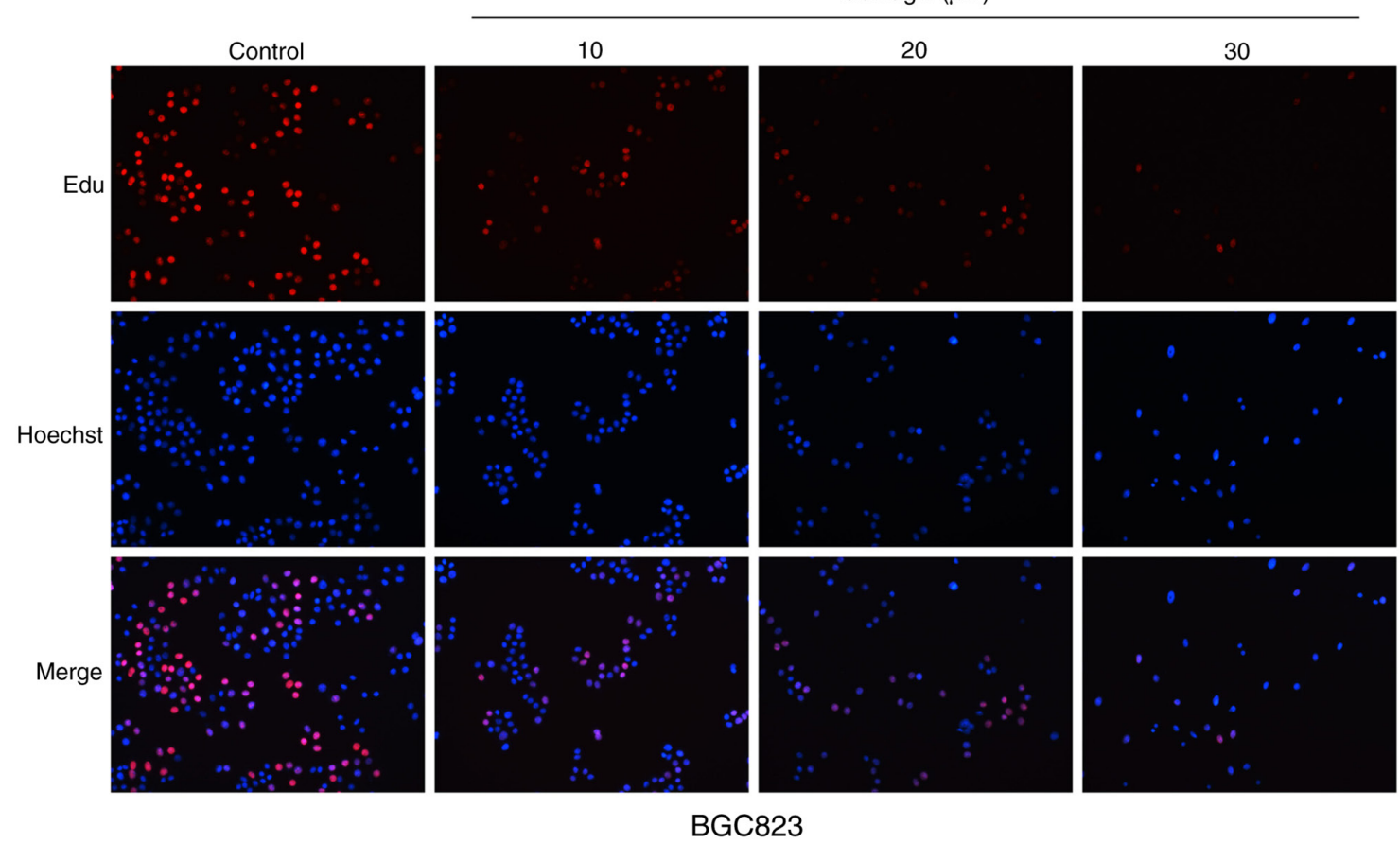

Figure 2. EdU assay to measure cell growth inhibition following corilagin treatment. (A) SGC7901 and (B) BGC823 cells were treated with various concentrations of corilagin $(0,10,20$ and $30 \mu \mathrm{M})$ for $24 \mathrm{~h}$, and then observed under a fluorescence microscope (magnification, $\mathrm{x} 200$ ).

blue fluorescence, which are characteristics of apoptotic-like morphological changes. The Annexin V/PI staining assay was performed to further detect the effect of corilagin on apoptosis. As shown in Fig. 3B, corilagin treatment induced apoptosis of the gastric cancer cells in a dose-dependent manner. Additionally, the number of early and late stage apoptotic cells were significantly increased following exposure to corilagin.

The release of LDH into culture media is one of the indicators of cell death (34). Therefore, the cytotoxic activity of corilagin on human gastric cancer cell lines was assessed using an LDH release assay. The results showed that the percentage of LDH release markedly increased in a concentration-dependent manner (Fig. 3C) and suggested that cell damage and cell death had occurred. To further investigate whether caspase-dependent apoptotic cell death had was present, Z-VAD-FMK, a pan caspase inhibitor, was used to detect cell viability using an MTT assay. As shown in Fig. 3D, Z-VAD-FMK markedly restored cell viability in the SGC7901 and BGC823 cell lines.

These results indicated that corilagin induced apoptotic cell death and that the caspase-dependent apoptotic pathway 
A
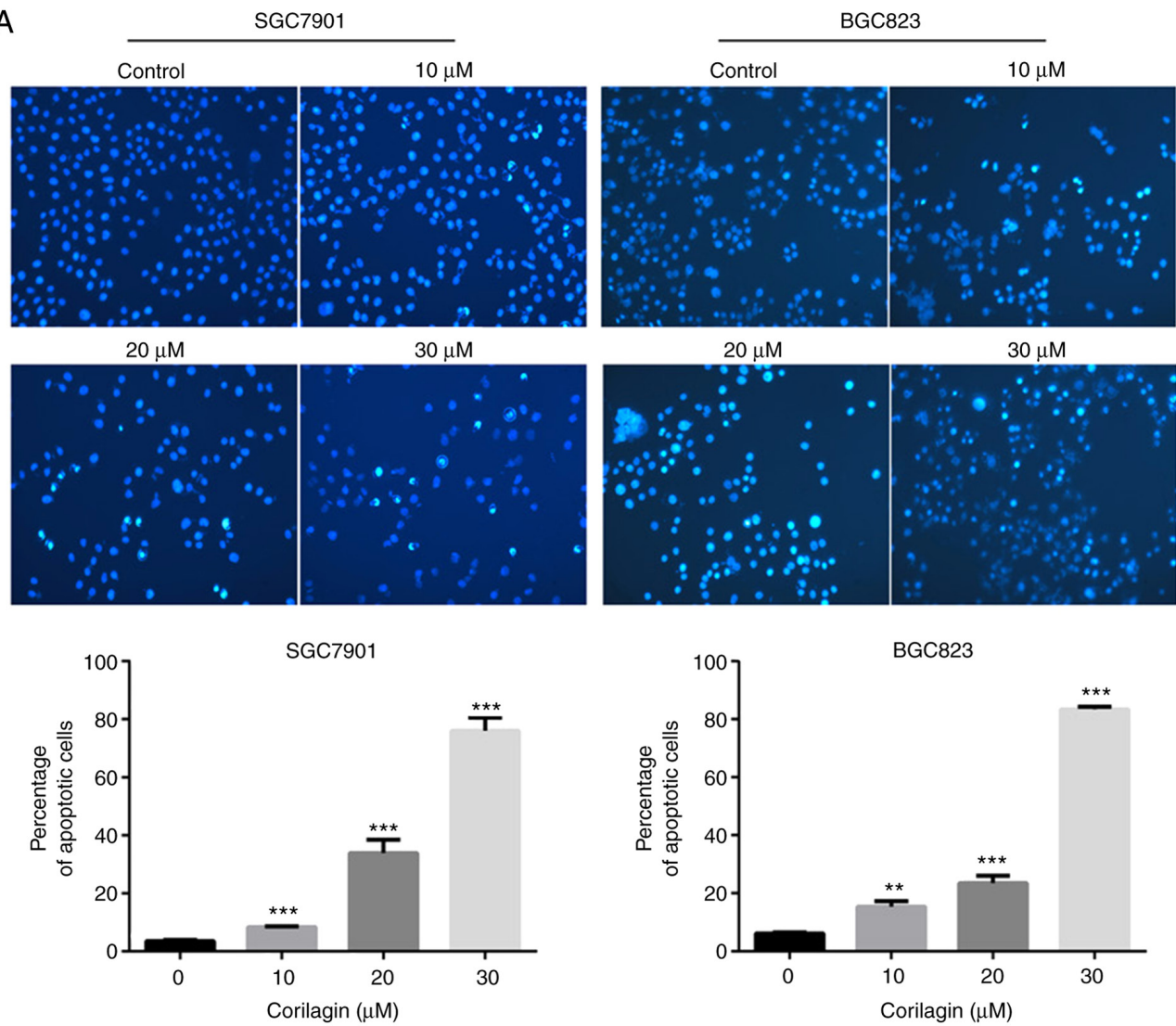

B

Corilagin (24 h)
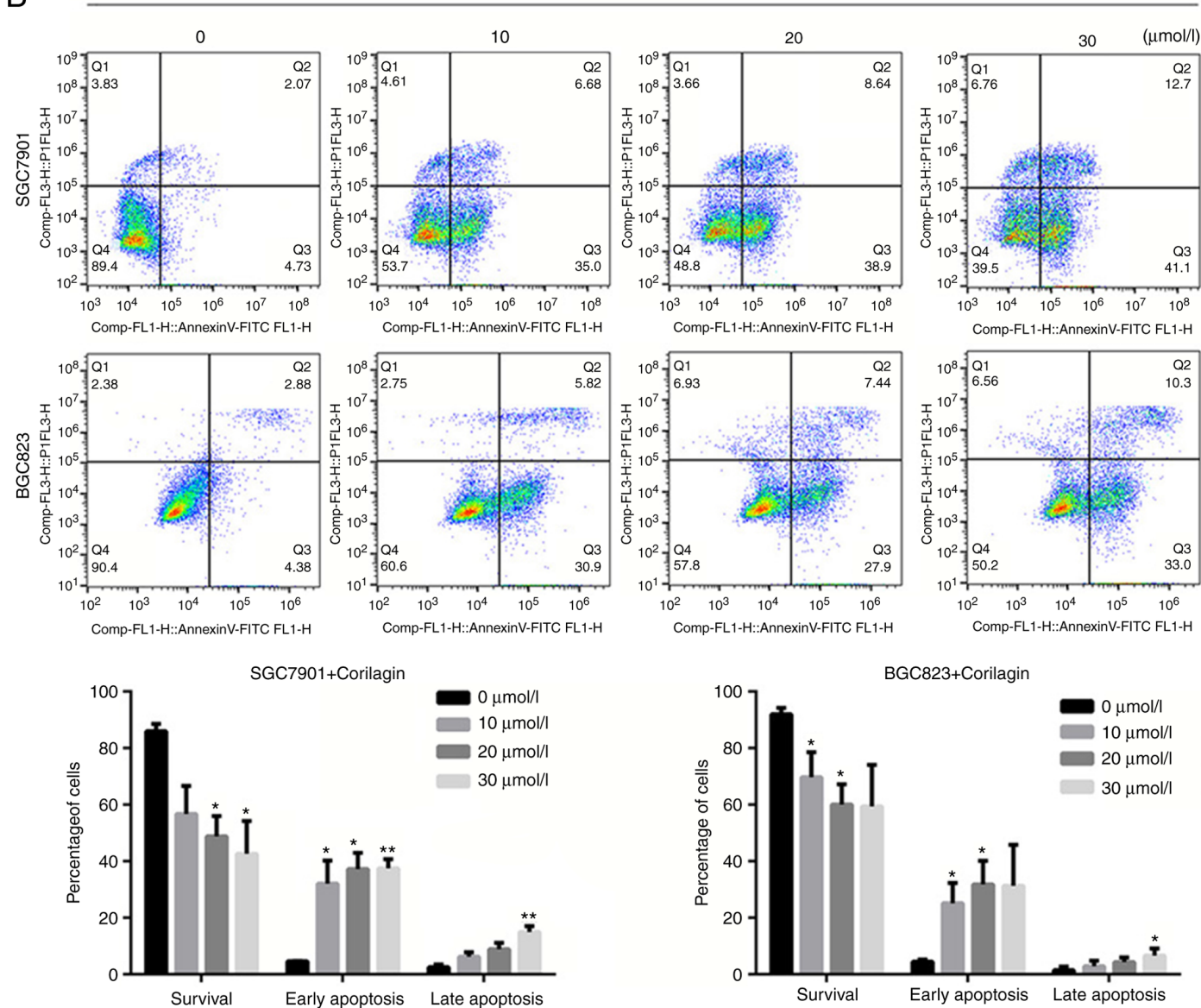

Figure 3. Corilagin induces apoptosis of human gastric cancer cells. (A) Cells were treated with various concentrations of corilagin $(0,10,20$ and $30 \mu \mathrm{M})$ for $24 \mathrm{~h}$, and then stained with Hoechst 33342. Images were captured by fluorescence microscopy at magnification, x200. Percentages of apoptotic cells are reported as the mean $\pm \mathrm{SD}(\mathrm{n}=3)$. (B) Corilagin-treated cells were analyzed by flow cytometry following staining with Annexin V-FITC and PI. The percentages of cells in survival, early and late apoptotic stages calculated and are expressed as the mean $\pm \mathrm{SD}(\mathrm{n}=3)$. 
C
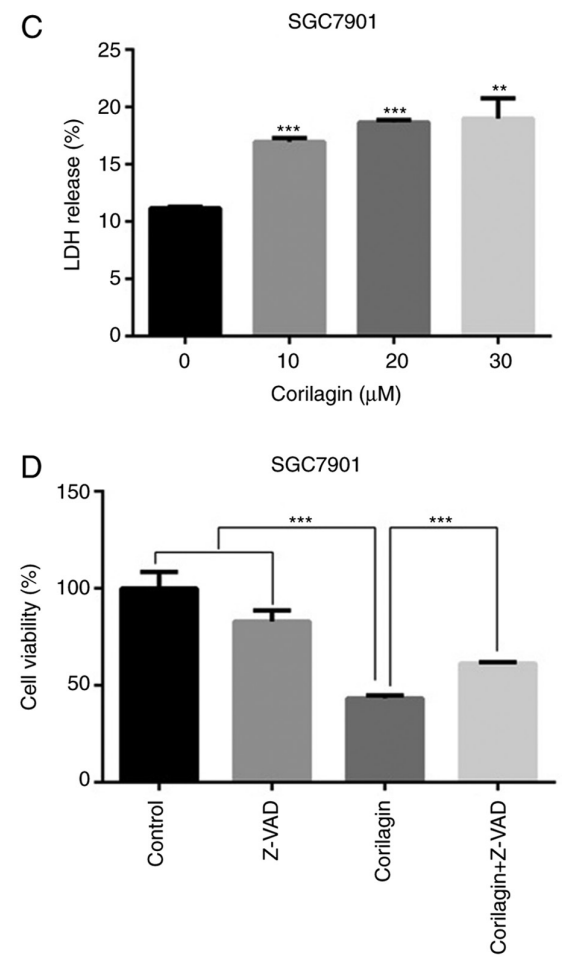
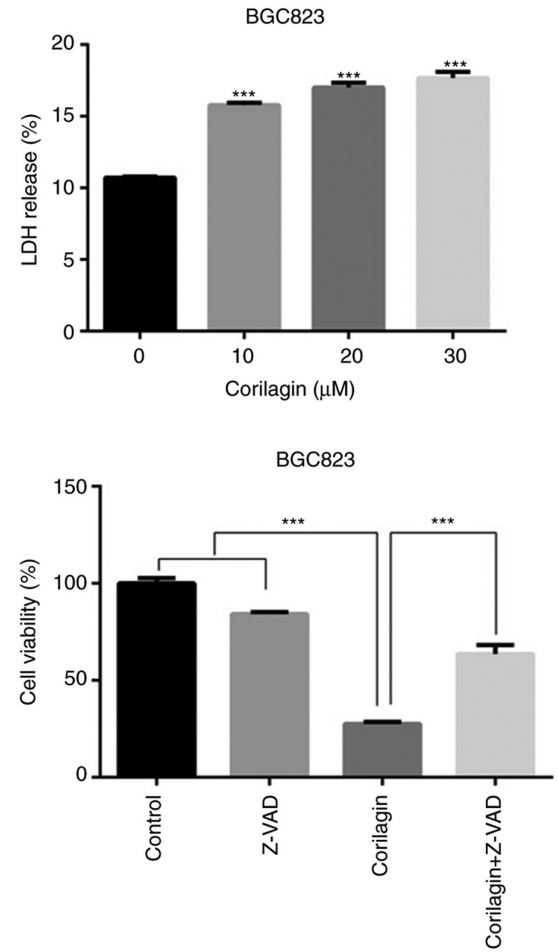

Figure 3. Continued. (C) LDH release from SGC7901 and BGC823 cells treated with corilagin for $24 \mathrm{~h}$. Values are reported as the mean \pm SD ( $=3$ ). (D) Cells were pretreated with $20 \mu \mathrm{M}$ Z-VAD-FMK for $2 \mathrm{~h}$ and then incubated with corilagin $(30 \mu \mathrm{M})$ for $24 \mathrm{~h}$. Cell viability was analyzed using a 3-(4,5-dimethylthiazol-2-yl)-2,5-diphenytetrazolium bromide assay. Data are reported as the mean $\pm \mathrm{SD}(\mathrm{n} \geq 3)$ of three replicate experiments. ${ }^{*} \mathrm{P}<0.05,{ }^{* *} \mathrm{P}<0.01$ and ${ }^{* * *} \mathrm{P}<0.001$ vs. control group (detected using Student's t-test). SD, standard deviation; PI, propidium iodide.

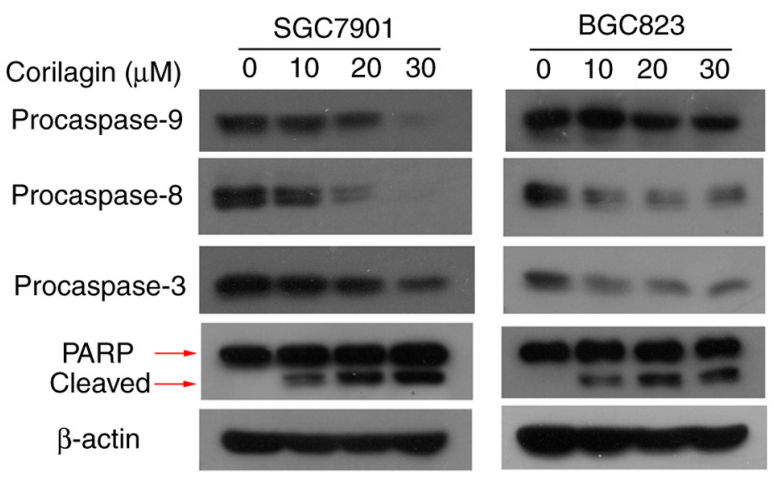

Figure 4. Expression levels of apoptosis-related proteins treated with corilagin in SGC7901 and BGC823 cells. Cells were treated with corilagin at various concentrations $(0,10,20$ and $30 \mu \mathrm{M})$ for $24 \mathrm{~h}$. Total protein was collected and the expression of caspases and PARP were analyzed by western blot assay. $\beta$-actin was used as an internal control. The data are representative of three replicate experiments. PARP, poly ADP-ribose polymerase.

may be key in the growth inhibitory effect of corilagin on gastric cancer cells.

Effect of corilagin on the expression of apoptosis-related proteins in human gastric cancer cells. To verify the mechanisms of apoptosis of human gastric cancer cells induced by corilagin, the expression of key proteins involved in cell apoptosis were detected by western blot analysis. As shown in Fig. 4, it was found that corilagin treatment decreased the protein levels of procaspase- $8,-9$ and -3 in a dose-dependent manner. Furthermore, corilagin increased the level of cleaved PARP in a concentration-dependent manner. These results suggested that corilagin induced caspase-dependent apoptosis of human gastric cancer cells.

Corilagin induces autophagy, which has a cytoprotective effect in human gastric cancer cells. To examine whether corilagin triggers the occurrence of autophagy, the formation of acidic autophagic vacuoles in gastric cancer cells was detected using AO dye. Under a fluorescence microscope, acidic vesicles exhibit bright red fluorescence and cell nuclei show green fluorescence. As shown in Fig. 5A, treatment with corilagin caused enhancement of acidic vesicles in SGC7901 and BGC823 cells compared with the control group (EBSS, as a positive control). It is known that LC3 protein is a key marker for autophagy. Therefore, LC3 conversion from LC3I to LC3II was assessed by western blot analysis. The results (Fig. 5B) showed that corilagin markedly increased the level of LC3II. These findings suggested that corilagin treatment induced autophagy of human gastric cancer cells.

To further corroborate whether autophagy has as protective effect on cancer cells, CQ, a late autophagy inhibitor, and corilagin were used to co-treat SGC7901 and BGC823 cells. The results from the western blot and MTT assays, respectively, showed that the expression of LC3II was markedly enhanced and cell viability was significantly decreased compared with the SGC7901 and BGC823 cells incubated in corilagin only (Fig. 5C and D). These data indicated that corilagin induced autophagy in gastric cancer cells and that the inhibition of autophagy improved the effect of corilagin on the suppression of cell proliferation.

Corilagin induces ROS generation in human gastric cancer cells. ROS, as significant signaling molecules, are involved in 
A
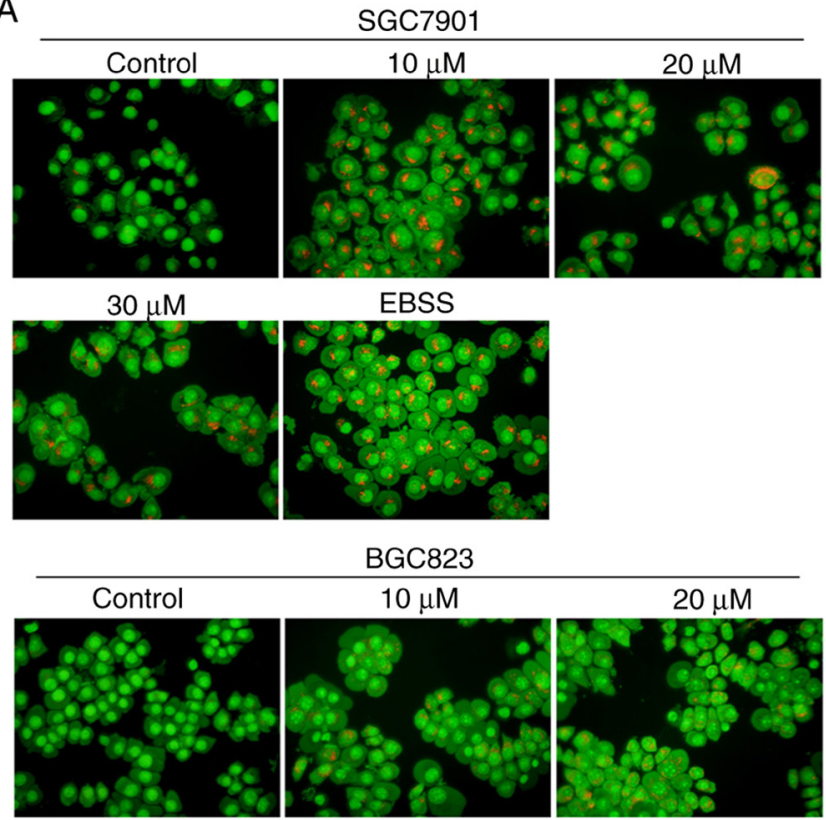

$30 \mu \mathrm{M}$

EBSS
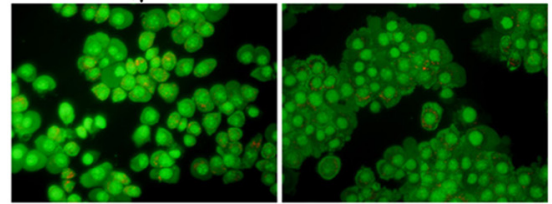

B
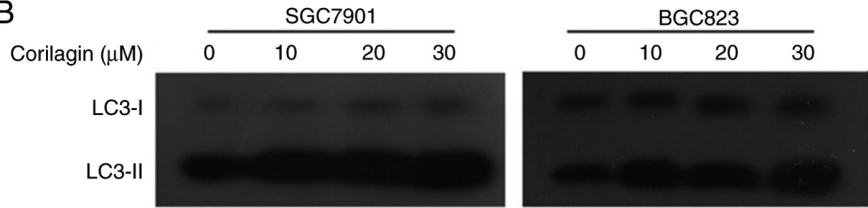

$\beta$-actin
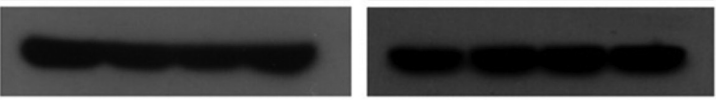

C
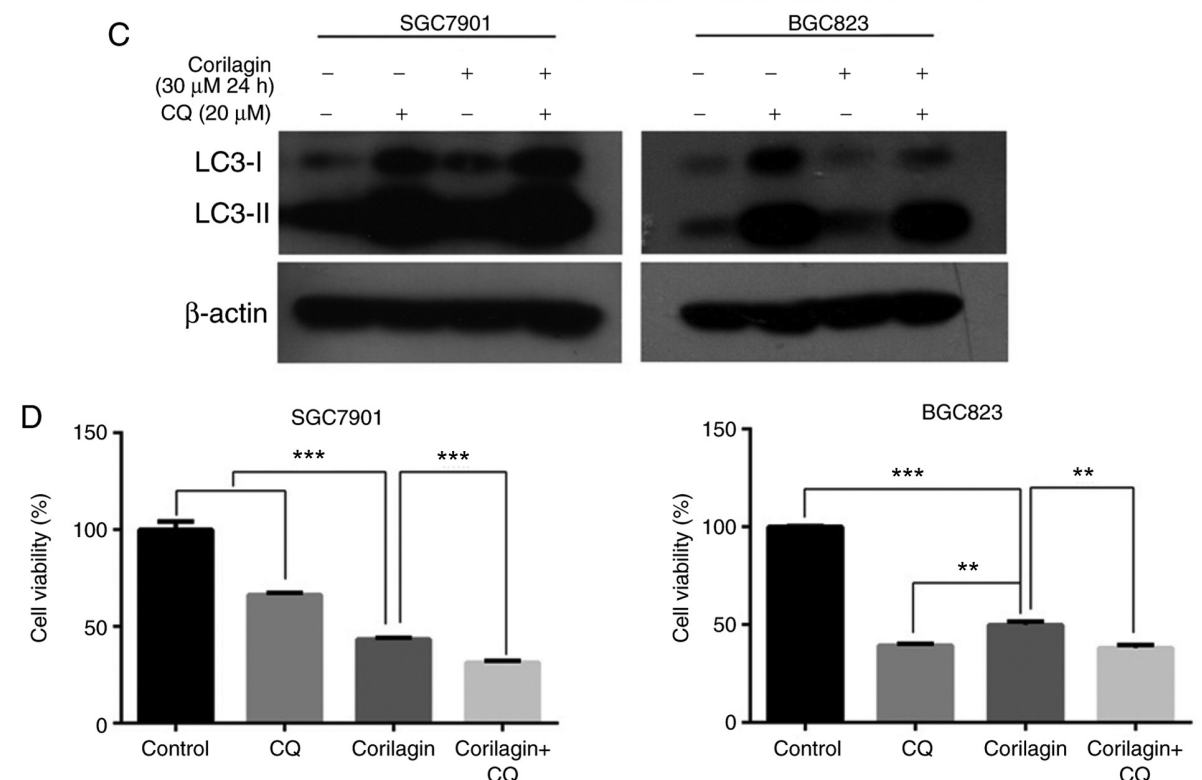

Figure 5. Corilagin triggers autophagy and inhibition of autophagy increases cell growth suppression in SGC7901 and BGC823 cells. (A) Observation of corilagin-induced acidic autophagic vesicles in SGC7901 and BGC823 cells using a fluorescence microscope. SGC7901 and BGC823 cells were treated with corilagin $(0,10,20$ and $30 \mu \mathrm{M})$ for $24 \mathrm{~h}$, followed by acridine orange dye (EBSS as positive control), and images were captured under a fluorescence microscope (magnification, x200). (B) Cells were incubated with corilagin at different concentrations $(0,10,20$ and $30 \mu \mathrm{M})$ for $24 \mathrm{~h}$, following which LC3I and LC3II levels were detected via western blot analysis. (C) SGC7901 and BGC823 cells were pretreated with CQ $(20 \mu \mathrm{M})$ for $2 \mathrm{~h}$, then incubated with corilagin (30 $\mu \mathrm{M})$ for $24 \mathrm{~h}$, and expression of LC3 was evaluated by western blot analysis. (D) Cells were pretreated with CQ $(20 \mu \mathrm{M})$ for $2 \mathrm{~h}$, then treated with corilagin ( $30 \mu \mathrm{M})$ for $24 \mathrm{~h}$, and cell viability was measured using a 3-(4,5-dimethylthiazol-2-yl)-2,5-diphenytetrazolium bromide assay. Results are expressed as the mean \pm standard deviation $(\mathrm{n} \geq 3)$ from three independent experiments. ${ }^{* *} \mathrm{P}<0.01$ and ${ }^{* * * *} \mathrm{P}<0.001$ vs. control group (detected using Student's $\mathrm{t}$-test). $\mathrm{CQ}$, chloroquine. 
signal transduction and sustaining cellular redox homeostasis in aerobic organisms (35). ROS are not only supporters of tumor cells, but are also efficient therapeutic tools in treating cancer (29). In the present study, intracellular ROS production was detected using a DCFH-DA probe in gastric cancer cells. Following treatment with corilagin for $24 \mathrm{~h}$, the flow cytometry revealed that corilagin significantly increased ROS generation in a dose-dependent manner (Fig. 6A). To further analyze the role of ROS, the ROS scavenger NAC was used to inhibit the accumulation of intracellular ROS (Fig. 6B). In addition, an MTT assay was performed to examine cell viability, as shown in Fig. 6C. Corilagin induced cell growth inhibition, which was markedly attenuated by pre-treatment with NAC. These data suggested that there is an increase of cell viability when the level of ROS was reduced with NAC.

Corilagin cannot activate necroptosis in SGC7901 and BGC823 cells. To identify whether corilagin induces necroptosis in gastric cancer cells, an MTT assay was used to examine the cell growth inhibitory effect of corilagin. First, the SGC7901 and BGC823 cells were pre-treated with necroptosis inhibitor Nec-1 or NSA for $2 \mathrm{~h}$, and then treated with corilagin for $24 \mathrm{~h}$. As shown in Fig. 7A and B, neither Nec-1 nor NSA significantly restored cell viability in the corilagin-treated SGC7901 and BGC823 cells. It is known that RIP3 is required for activation of the necroptosis signaling pathway. Therefore, RT-PCR and western blot analyses were performed to examine the expression of RIP3. The results, as shown in Fig. 7C and D, revealed that RIP3 was not expressed in the SGC7901 or BGC823 cells. HeLa cells were used as a negative control and HT-29 cells were used as a positive control. These results demonstrated that corilagin cannot induce necroptosis in SGC7901 and BGC823 cell lines.

\section{Discussion}

As far as we know, although certain pharmacological and biochemical effects of corilagin have been reported, the anticancer effect of corilagin has received limited investigation. Attar et al (18) investigated the apoptotic and genomic effects of corilagin on SKOV3 ovarian carcinoma cells. Their results indicated that corilagin increased apoptosis and altered the genomic expression levels in SKOV3 cells in a time- and dose-dependent manner. However, there have been no reports on the effect of corilagin in gastric carcinoma cells. In the present study, it was first exhibited that corilagin markedly inhibited cell proliferation in a concentration-dependent manner in SGC7901 and BGC823 cells. Furthermore, it was demonstrated that corilagin showed less toxicity towards normal cells, for example, GES-1 human gastric mucosal epithelial cells. These results indicated that corilagin has potent antitumor activity against gastric cancer cells with less toxicity towards normal cells.

To the best of our knowledge, apoptosis is a specific type of programmed cell death that is key in the antitumor activity of several natural products. In the present study, it was shown that the growth inhibitory properties of corilagin were due to apoptotic cell death, as detected by Annexin V/PI staining. Additionally, it was also found that the gastric cancer cells exhibited the characteristics of apoptotic-like morphological changes, determined using a Hoechst 33342 staining assay. These data suggested that corilagin induces the apoptosis of human gastric cancer cells. Caspases have been widely investigated in the past (36); they are key in the cascade that result in apoptosis. Caspases are classified either as initiators or executors of apoptosis, depending on their time of entry into the cell death process. Initiator caspases, including caspases $-2,-8$, -9 and -10 , enter the apoptotic cascade in the early stage and are responsible for activating the executor caspases $-3,-6$ or -7 (37). In the present study, it was shown that the protein levels of procaspase $-8,-9$ and -3 were decreased following corilagin treatment. Simultaneously, PARP, an indicator of caspase activation, was cleaved by corilagin incubation. The expression level of cleaved PARP was increased in a dose-dependent manner in SGC7901 and BGC823 cells. It was also found that Z-VAD-FMK markedly restored cell viability. Taken together, these data certified that corilagin induced caspase-dependent apoptotic cell death in human gastric cancer.

As a specific cell death pathway, autophagy can be triggered by various intracellular stimuli and it has conflicting effects in tumor therapy, as it can have tumor-promoting or tumor-inhibiting effects. The role of autophagy in diverse therapies is cell line- and activator-dependent (38). In the present study, the activation of autophagy was observed in corilagin-treated SGC7901 and BGC823 cells. The autophagy inhibitor, CQ, was used to detect whether autophagy has a protective effect on corilagin-exposed gastric cancer cells. The results showed that CQ and corilagin co-treatment further decreased cell viability compared with that in cells treated with corilagin alone. Accordingly, it was hypothesized that corilagin induces autophagy, which has a cytoprotective role in human gastric cancer cells. However, additional data are required to confirm this conclusion.

ROS is a collective noun for the reactive forms of oxygen, and acts as a second messenger that is involved in signaling cascades (39). The excessive generation of ROS can result in oxidative stress, DNA damage and cell death through apoptosis or necrosis (40). For example, Su et al (41) found that sonodynamic treatment significantly increased intracellular ROS generation and induced the apoptosis of K562 human leukemia cells in a ROS-dependent manner. Similarly, in the present study, it was shown that corilagin markedly increased ROS production in the SGC7901 and BGC823 cells. The present study also demonstrated that the cell growth inhibition induced by corilagin was effectively attenuated by pre-treatment with NAC. These results suggested that ROS may be vital in the inhibition of human gastric cancer cell growth caused by corilagin treatment.

Based on the above results, it was confirmed that corilagin can induce autophagy, apoptosis and ROS accumulation in gastric cancer cells. The specific associations among these factors have attracted increased attention. A study by Srivastava et al (42) reported that neem oil limonoids triggered p53-independent apoptosis and autophagy in cancer cells; furthermore, they found the existence of cross-talk between them. Numerous studies have demonstrated that autophagy has as a protective role in several types of cancer cell and the inhibition of autophagy can increase anticancer drug-induced apoptosis $(43,44)$. In addition, ROS have been 

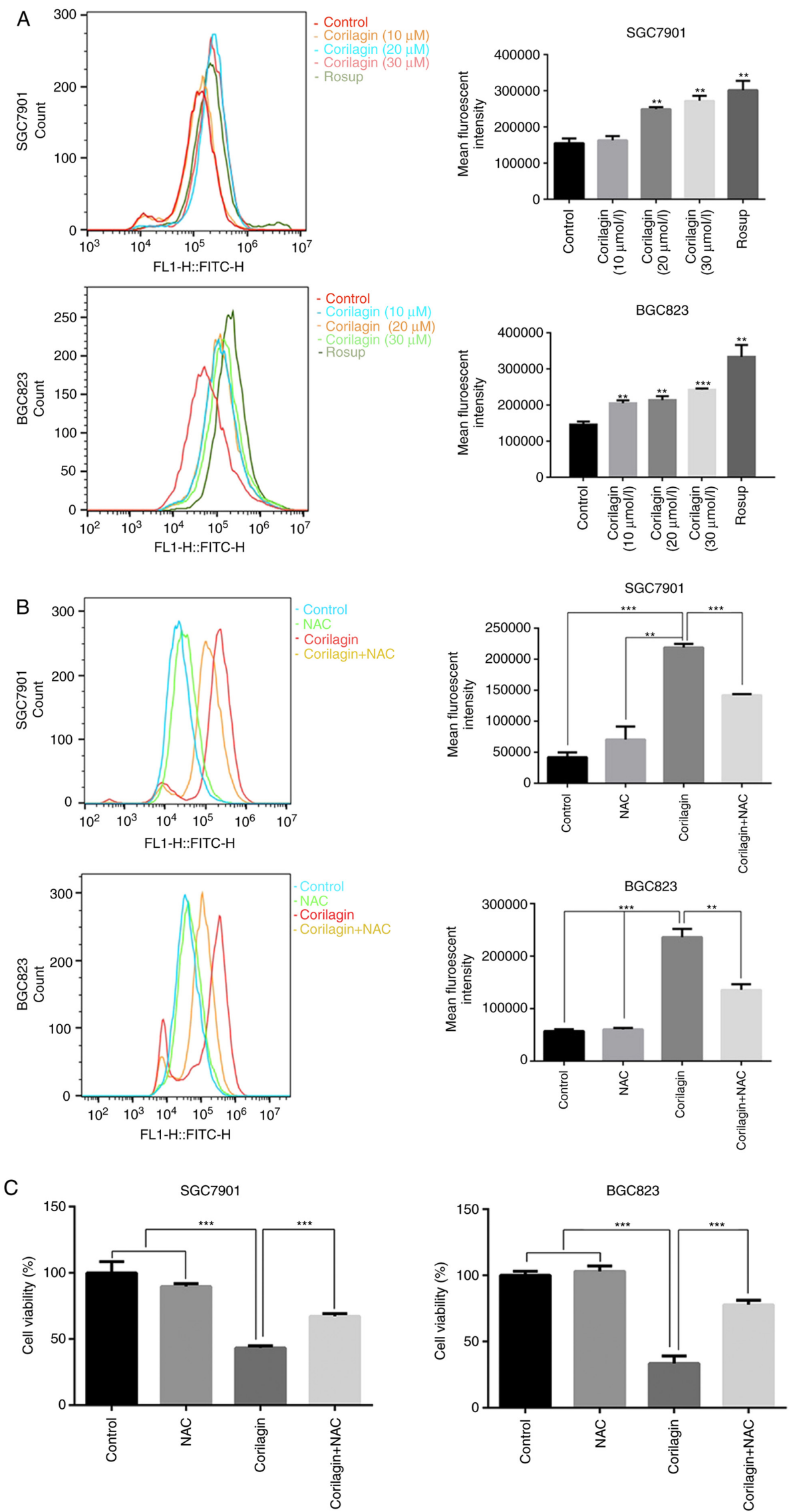

Figure 6. Corilagin triggers ROS generation in gastric cancer cells. (A) Cells were treated with corilagin at various concentrations $(0,10,20$ and $30 \mu \mathrm{M})$ for $24 \mathrm{~h}$ and then labeled with DCFH-DA $(10 \mu \mathrm{M})$. ROS production were measured by flow cytometry (Rosup as positive control). (B) Pre-treatment with NAC $(5 \mu \mathrm{M})$ for $2 \mathrm{~h}$ and then incubated with corilagin $(30 \mu \mathrm{M})$ for $24 \mathrm{~h}$. Cells were stained with DCFH-DA $(10 \mu \mathrm{M})$ and then ROS levels were determined using flow cytometry. (C) Cells were pretreated with NAC $(5 \mu \mathrm{M})$ and then exposed to corilagin $(30 \mu \mathrm{M})$ for $24 \mathrm{~h}$. Following treatment, the cell viabilities were detected using a 3-(4,5-dimethylthiazol-2-yl)-2,5-diphenytetrazolium bromide assay. The data are represented as the mean \pm standard deviation ( $\mathrm{n}=3$ ) of three replicate experiments. ${ }^{* *} \mathrm{P}<0.01$ and ${ }^{* * *} \mathrm{P}<0.001$ vs. control group (detected using Student's t-test). ROS, reactive oxygen species; NAC, N-acetyl-L-cysteine. 
A

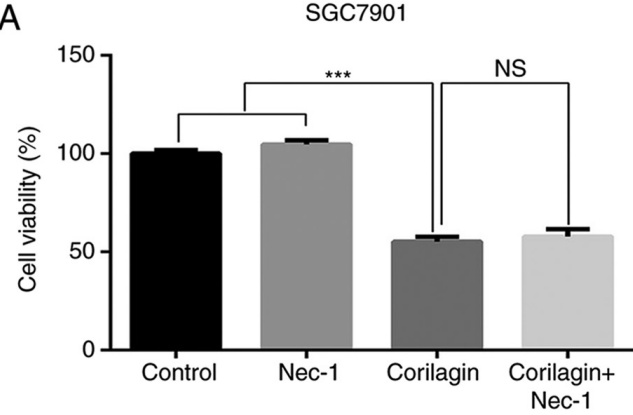

B

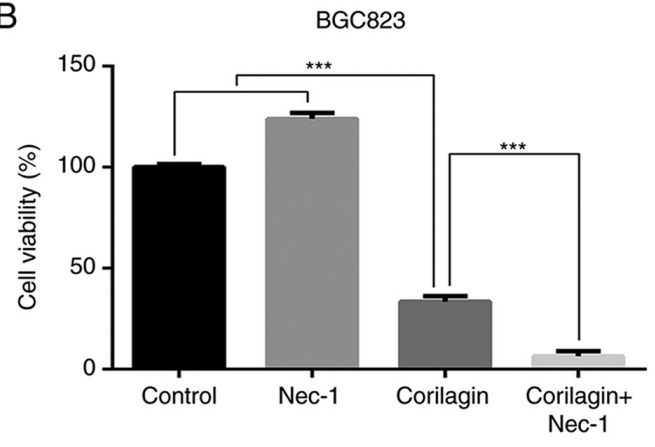

C

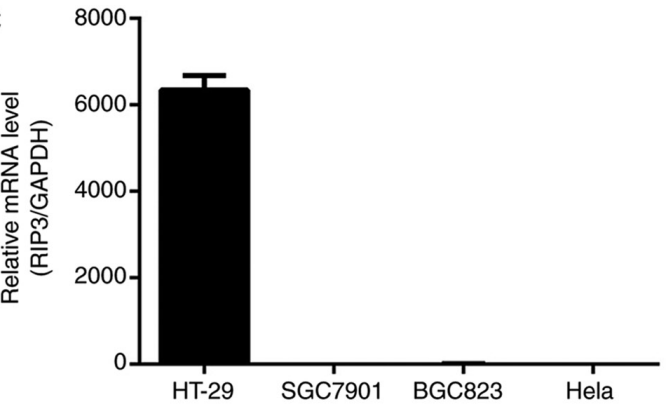

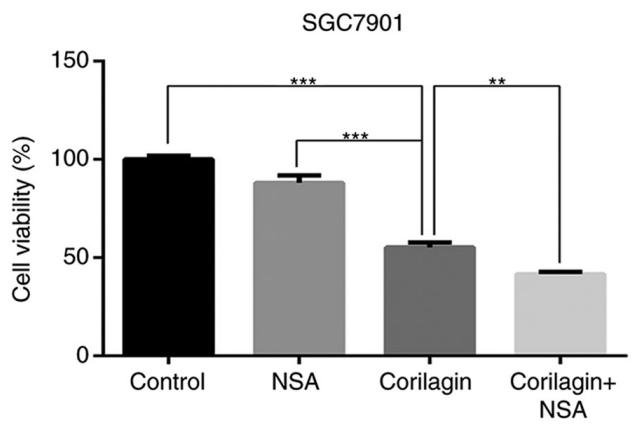

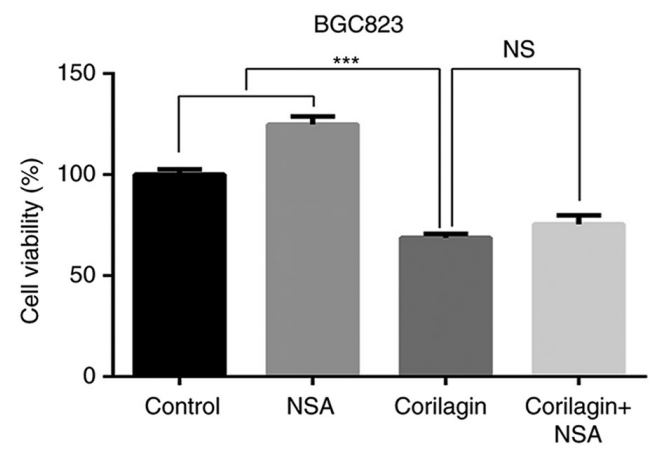

D

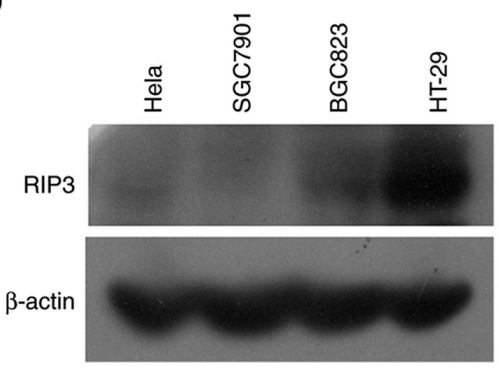

Figure 7. Corilagin cannot trigger necroptosis in SGC7901 and BGC823 cells. (A) SGC7901 and (B) BGC823 cells were pre-treated with necroptosis inhibitor Nec-1 $(20 \mu \mathrm{M})$ or NSA $(20 \mathrm{nM})$ for $2 \mathrm{~h}$, and then treated with corilagin $(30 \mu \mathrm{M})$ for $24 \mathrm{~h}$. The cell viabilities were then examined using a 3-(4,5-dimethylthiazol-2-yl)-2,5-diphenytetrazolium bromide assay. Levels of RIP3 were measured by (C) reverse transcription-polymerase chain reaction and (D) western blot analyses in several cancer cell lines. The data are reported as the mean \pm standard deviation ( $\mathrm{n}=3$ ) of three replicate experiments. ${ }^{* *} \mathrm{P}<0.01$ and ${ }^{* * * *} \mathrm{P}<0.001$ vs. control group (detected using Student's t-test). RIP3, receptor interaction protein 3; Nec-1, necrostatin-1; NSA, necro-sulfonamide; ns, not significant.

shown to be important in the pathways of cellular apoptosis and autophagy (45). For example, Xu et al (46) demonstrated that the inhibition of autophagy increased ROS-mediated apoptosis in mesangial cells. They revealed that apoptosis and autophagy were dependent on ROS production. In the present study, it was suggested that the inhibition of autophagy enhanced the effect of corilagin on gastric cancer cells. Therefore, autophagy may inhibit apoptosis in corilagin-treated SGC7901 and BGC823 cells. In addition, corilagin markedly enhanced ROS generation, which is vital in inhibiting the proliferation of human gastric cancer cells. Therefore, it is possible that ROS accumulation promoted apoptosis of the corilagin-exposed gastric cancer cells. However, further examination is required to analyze the associations of apoptosis, autophagy and ROS accumulation in subsequent investigations.

Necroptosis, namely programmed necrosis, is identified as an emerging form of programmed cell death distinct from apoptosis. Necroptosis can be activated by several inducements, for example one of which stimulates TNF- $\alpha$ and is a typical response. In TNF- $\alpha$-induced necroptosis, RIP3 is necessary as a key regulator (47). In the present study, it was initially verified that cell viability was not enhanced via pre-treatment with necroptosis inhibitor Nec-1 or NSA prior to corilagin treatment in SGC7901 and BGC823 cells. It was then shown that RIP3 was not expressed in the SGC7901 and BGC823 cell lines. Therefore, these findings suggest that corilagin cannot induce necroptosis in human gastric cancer cells, but further investigations are required to confirm this.

In conclusion, corilagin can distinctly inhibit the proliferation of SGC7901 and BGC823 cells in vitro, while showing low toxicity towards normal cells. Furthermore, corilagin-treated cells showed induction of apoptotic cell death by activating caspase $-8,-9,-3$ and PARP proteins. Alternatively, corilagin-induced the autophagy of gastric cancer cells and the inhibition of autophagy improved the effects of corilagin on cell growth suppression. The excessive production of ROS may be important in inhibiting the growth of gastric cancer cells following corilagin treatment. The novel cell death pathway, necroptosis, was not induced by corilagin-incubation in the SGC7901 and BGC823 cell lines. Therefore, the natural product, corilagin may be a promising novel drug for the treatment of human gastric cancer. 


\section{Acknowledgements}

The authors would like to thank Professor Tianhui Hu (Cancer Research Center, Medical College of Xiamen University, Xiamen, China) for supporting this study. The authors would also like to thank the valuable suggestions and comments on this article received from editor and reviewers.

\section{Funding}

The present study was supported by the grants from the National Natural Science Foundation of China (grant nos. 81872045 and 81470793), the Special Fund for Public Welfare Research Institutes of Fujian Province (grant no. 2017R1036-1 and 2018R1036-4) and the Major Technology Program of Industry-University-Research Cooperation in Fujian Province (grant no. 2016Y4011).

\section{Availability of data and materials}

The datasets used and/or analysed during the current study are available from the corresponding author on reasonable request.

\section{Authors' contributions}

JX conceived and performed the experiments and wrote the manuscript. JX, GZ, YT and JY analysed the data. YL and GS designed the study and provided funding. All authors read and approved the final manuscript.

\section{Ethics approval and consent to participate}

Not applicable.

\section{Patient consent for publication}

Not applicable.

\section{Competing interests}

The authors declare that they have no competing interests.

\section{References}

1. Wang C, Zhang J, Cai M, Zhu Z, Gu W, Yu Y and Zhang X: DBGC: A database of human gastric cancer. PLoS One 10 e142591, 2015.

2. Torre LA, Bray F, Siegel RL, Ferlay J, Lortet-Tieulent J and Jemal A: Global cancer statistics, 2012. CA Cancer J Clin 65: 87-108, 2015.

3. Hu C, Song G, Zhang B, Liu Z, Chen R, Zhang H and Hu T: Intestinal metabolite compound $K$ of panaxoside inhibits the growth of gastric carcinoma by augmenting apoptosis via Bid-mediated mitochondrial pathway. J Cell Mol Med 16: 96-106, 2012.

4. Parkin DM, Bray F, Ferlay J and Pisani P: Global cancer statistics, 2002. CA Cancer J Clin 55: 74-108, 2005.

5. Kodera Y, Fujiwara M, Koike M and Nakao A: Chemotherapy as a component of multimodal therapy for gastric carcinoma. World J Gastroenterol 12: 2000-2005, 2006.

6. Ji YB, Qu ZY and Zou X: Juglone-induced apoptosis in human gastric cancer SGC-7901 cells via the mitochondrial pathway. Exp Toxicol Pathol 63: 69-78, 2011.

7. Dai ZJ, Gao J, Ji ZZ, Wang XJ, Ren HT, Liu XX, Wu WY, Kang HF and Guan HT: Matrine induces apoptosis in gastric carcinoma cells via alteration of Fas/FasL and activation of caspase-3. J Ethnopharmacol 123: 91-96, 2009.
8. Duan W, Yu Y and Zhang L: Antiatherogenic effects of phyllanthus emblica associated with corilagin and its analogue. Yakugaku Zasshi 125: 587-591, 2005.

9. Rangkadilok N, Worasuttayangkurn L, Bennett RN and Satayavivad J: Identification and quantification of polyphenolic compounds in Longan (Euphoria longana Lam.) fruit. J Agric Food Chem 53: 1387-1392, 2005.

10. Darwish AG, Samy MN, Sugimoto S, Otsuka H, Abdel-Salam H and Matsunami K: Effects of hepatoprotective compounds from the leaves of Lumnitzera racemosa on acetaminophen-induced liver damage in vitro. Chem Pharm Bull (Tokyo) 64: 360-365, 2016.

11. Kinoshita S, Inoue Y, Nakama S, Ichiba T and Aniya Y: Antioxidant and hepatoprotective actions of medicinal herb, Terminalia catappa L. from Okinawa Island and its tannin corilagin. Phytomedicine 14: 755-762, 2007.

12. Notka F, Meier GR and Wagner R: Inhibition of wild-type human immunodeficiency virus and reverse transcriptase inhibitor-resistant variants by Phyllanthus amarus. Antiviral Res 58: 175-186, 2003.

13. Zhao L, Zhang SL, Tao JY, Pang R, Jin F, Guo YJ, Dong JH, Ye P, Zhao HY and Zheng GH: Preliminary exploration on anti-inflammatory mechanism of Corilagin (beta-1-O-galloyl-3,6-(R)-hexahydroxydiphenoyl-D-glucose) in vitro. Int Immunopharmacol 8: 1059-1064, 2008.

14. Guo YJ, Luo T, Wu F, Liu H, Li HR, Mei YW, Zhang SL, Tao JY, Dong JH, Fang Y and Zhao L: Corilagin protects against HSV1 encephalitis through inhibiting the TLR2 signaling pathways in vivo and in vitro. Mol Neurobiol 52: 1547-1560, 2015.

15. Liu FC, Chaudry IH and Yu HP: Hepatoprotective effects of Corilagin following hemorrhagic shock are through Akt-dependent pathway. Shock 47: 346-351, 2017.

16. Du P, Ma Q, Zhu ZD, Li G, Wang Y, Li QQ, Chen YF, Shang ZZ, Zhang $\mathrm{J}$ and Zhao L: Mechanism of Corilagin interference with IL-13/STAT6 signaling pathways in hepatic alternative activation macrophages in schistosomiasis-induced liver fibrosis in mouse model. Eur J Pharmacol 793: 119-126, 2016.

17. Ming Y, Zheng Z, Chen L, Zheng G, Liu S, Yu Y and Tong Q: Corilagin inhibits hepatocellular carcinoma cell proliferation by inducing G2/M phase arrest. Cell Biol Int 37: 1046-1054, 2013.

18. Attar R, Cincin ZB, Bireller ES and Cakmakoglu B: Apoptotic and genomic effects of corilagin on SKOV3 ovarian cancer cell line. Onco Targets Ther 10: 1941-1946, 2017.

19. Gu Y, Xiao L, Ming Y, Zheng Z and Li W: Corilagin suppresses cholangiocarcinoma progression through Notch signaling pathway in vitro and in vivo. Int J Oncol 48: 1868-1876, 2016.

20. Milani R,BrognaraE, Fabbri E,Finotti A,Borgatti M,Lampronti I, Marzaro G, Chilin A, Lee KK, Kok SH, et al: Corilagin induces high levels of apoptosis in the Temozolomide-resistant T98G glioma cell line. Oncol Res, 2017.

21. Jia L, Jin H, Zhou J, Chen L, Lu Y, Ming Y and Yu Y: A potential anti-tumor herbal medicine, Corilagin, inhibits ovarian cancer cell growth through blocking the TGF- $\beta$ signaling pathways. BMC Complement Altern Med 13: 33, 2013.

22. Li X, Deng Y, Zheng Z, Huang W, Chen L, Tong Q and Ming Y: Corilagin, a promising medicinal herbal agent. Biomed Pharmacother 99: 43-50, 2018.

23. Hikita H, Kodama T, Shimizu S, Li W, Shigekawa M, Tanaka S, Hosui A, Miyagi T, Tatsumi T, Kanto T, et al: Bak deficiency inhibits liver carcinogenesis: A causal link between apoptosis and carcinogenesis. J Hepatol 57: 92-100, 2012.

24. Ahamed M, Akhtar MJ, Siddiqui MA, Ahmad J, Musarrat J, Al-Khedhairy AA, AlSalhi MS and Alrokayan SA: Oxidative stress mediated apoptosis induced by nickel ferrite nanoparticles in cultured A549 cells. Toxicology 283: 101-108, 2011.

25. Zhang S, Li T, Zhang L, Wang X, Dong H, Li L, Fu D, Li Y, Zi X, Liu HM, et al: A novel chalcone derivative S17 induces apoptosis through ROS dependent DR5 up-regulation in gastric cancer cells. Sci Rep 7: 9873, 2017.

26. Shimizu S, Yoshida T, Tsujioka M and Arakawa S: Autophagic cell death and cancer. Int J Mol Sci 15: 3145-3153, 2014.

27. Xu B, Xu M, Tian Y, Yu Q, Zhao Y, Chen X, Mi P, Cao H, Zhang B, Song G, et al: Matrine induces RIP3-dependent necroptosis in cholangiocarcinoma cells. Cell Death Discov 3: $16096,2017$.

28. Wu W, Liu P and Li J: Necroptosis: An emerging form of programmed cell death. Crit Rev Oncol Hematol 82: 249-258, 2012.

29. Manda G, Isvoranu G, Comanescu MV, Manea A, Debelec Butuner B and Korkmaz KS: The redox biology network in cancer pathophysiology and therapeutics. Redox Biol 5: 347-357, 2015. 
30. Chen H, Li Y, Zhu Y, Wu L, Meng J, Lin N, Yang D, Li M, Ding W, Tong X and Su Q: Advanced glycation end products promote ChREBP expression and cell proliferation in liver cancer cells by increasing reactive oxygen species. Medicine (Baltimore) 96: e7456, 2017.

31. Lee Y, Sung B, Kang YJ, Kim DH, Jang JY, Hwang SY, Kim M, Lim HS, Yoon JH, Chung HY and Kim ND: Apigenin-induced apoptosis is enhanced by inhibition of autophagy formation in HCT116 human colon cancer cells. Int J Oncol 44: 1599-1606, 2014.

32. Huang $\mathrm{P}$, Zhang $\mathrm{YH}$, Zheng XW, Liu YJ, Zhang H, Fang L, Zhang YW, Yang C, Islam K, Wang $C$ and Naranmandura $H$ : Phenylarsine oxide (PAO) induces apoptosis in HepG2 cells via ROS-mediated mitochondria and ER-stress dependent signaling pathways. Metallomics 9: 1756-1764, 2017.

33. Livak KJ and Schmittgen TD: Analysis of relative gene expression data using real-time quantitative PCR and the 2(-Delta Delta C(T)) method. Methods 25: 402-408, 2001.

34. Attarde SS and Pandit SV: Cytotoxic activity of NN-32 toxin from Indian spectacled cobra venom on human breast cancer cell lines. BMC Complement Altern Med 17: 503, 2017.

35. Briehl MM: Oxygen in human health from life to death-An approach to teaching redox biology and signaling to graduate and medical students. Redox Biol 5: 124-139, 2015.

36. Shalini S, Dorstyn L, Dawar S and Kumar S: Old, new and emerging functions of caspases. Cell Death Differ 22: 526-539, 2015.

37. MacKenzie SH and Clark AC: Death by caspase dimerization. Adv Exp Med Biol 747: 55-73, 2012.

38. Zhao C, She T, Wang L, Su Y, Qu L, Gao Y, Xu S, Cai S and Shou C: Daucosterol inhibits cancer cell proliferation by inducing autophagy through reactive oxygen species-dependent manner. Life Sci 137: 37-43, 2015.

39. Forman HJ, Maiorino M and Ursini F: Signaling functions of reactive oxygen species. Biochemistry 49: 835-842, 2010.
40. Eckert A, Keil U, Marques CA, Bonert A, Frey C, Schüssel K and Müller WE: Mitochondrial dysfunction, apoptotic cell death, and Alzheimer's disease. Biochem Pharmacol 66: 1627-1634, 2003.

41. Su X, Wang P, Yang S, Zhang K, Liu Q and Wang X: Sonodynamic therapy induces the interplay between apoptosis and autophagy in K562 cells through ROS. Int J Biochem Cell Biol 60: 82-92, 2015.

42. Srivastava P, Yadav N, Lella R, Schneider A, Jones A, Marlowe T, Lovett G, O'Loughlin K, Minderman H, Gogada R and Chandra D: Neem oil limonoids induces p53-independent apoptosis and autophagy. Carcinogenesis 33: 2199-2207, 2012.

43. Masud AM, Kariya R, Kawaguchi A, Matsuda K, Kudo E and Okada S: Inhibition of autophagy by chloroquine induces apoptosis in primary effusion lymphoma in vitro and in vivo through induction of endoplasmic reticulum stress. Apoptosis 21: 1191-1201, 2016.

44. Wang X, Wang P, Zhang K, Su X, Hou J and Liu Q: Initiation of autophagy and apoptosis by sonodynamic therapy in murine leukemia L1210 cells. Toxicol In Vitro 27: 1247-1259, 2013.

45. Datta K, Babbar P, Srivastava T, Sinha S and Chattopadhyay P: p53 dependent apoptosis in glioma cell lines in response to hydrogen peroxide induced oxidative stress. Int J Biochem Cell Biol 34: 148-157, 2002.

46. Xu L, Fan Q, Wang X, Zhao X and Wang L: Inhibition of autophagy increased AGE/ROS-mediated apoptosis in mesangial cells. Cell Death Dis 7: e2445, 2016.

47. Liu X, Li Y, Peng S, Yu X, Li W, Shi F, Luo X, Tang M, Tan Z, Bode AM and Cao Y: Epstein-Barr virus encoded latent membrane protein 1 suppresses necroptosis through targeting RIPK1/3 ubiquitination. Cell Death Dis 9: 53, 2018.

This work is licensed under a Creative Commons Attribution-NonCommercial-NoDerivatives 4.0 International (CC BY-NC-ND 4.0) License. 